\title{
INFLUENCE OF THE CROSS SECTION SHAPE ON THE ION RELEASE AND SUBSEQUENT WEIGHT CHANGE OF DIFFERENT ORTHODONTIC ALLOYS
}

\author{
Nasser A. M. Barakat ${ }^{1}$, Wael M Refai ${ }^{2}$, \\ Ibrahim H. Mahmoud ${ }^{3}$, Hak Yong Kim ${ }^{4}$
}

ABSTRACT:

In this study, effect of the cross section shape on the ion release of different orthodontic alloys as well as subsequent weight changes was investigated. In vitro experiments were carried out on three different alloys: stainless steel, nickel-titanium, copper-titanium alloys. Both round and rectangular wires were utilized. The results revealed that the cross section did not have considerable impact. In case of stainless steel and nickel-titanium alloys, the round cross section revealed high ion release. However, in case of nickelcopper-titanium alloy, the rectangular cross sectional wires showed more ion release. Concerning weight changes, stainless steel wire showed more weight increase. Conversely, rectangular copper titanium wire showed weight loss.

Key Words: Orthodontic wires, corrosion in orthodontics, cross section effect.

1- Ass Prof of Organic Materials and Fiber Engineering, Organic Materials and Fiber Engineering Dept, Chonbuk National University, Jeonju 561-756, Republic of South Korea.

2- Prof of Chemical Engineering, Chemical Engineering Dept, Faculty of Engineering, Minia University, El Minia, Egypt.

3- Ass Prof of Orthodontics, Orthodontic Dept, Faculty of Dentistry, Minia University, El Minia, Egypt.

4- Prof of Organic Materials and Fiber Engineering, Organic Materials and Fiber Engineering Dept. Organic Materials and Fiber Engineering Dept. Chonbuk National University Jeonju, 561-756 South Korea. 
Egyptian

Orthodontic Journal

\section{INTRODUCTION}

A considerable increase in orthodontic treatment has drawn the attention to potential undesirable effects. The most common alloys used in orthopedics and dentistry are stainless steel, nickel-titanium and nickel-copper-titanium alloys. ${ }^{(1)}$

Electrolytic or electrochemical corrosion occurs in the oral cavity due to the wet environment. A metal in aqueous solution will be thermodynamically unstable if its tendency to pass from solid state to the ionic form is associated with a decrease in energy. The direction of energy change is influenced by factors such as the metal itself, shape and phase of the metal, galvanic coupling of dissimilar metals, solution composition, $\mathrm{pH}$, and temperature. If the metal is unstable, it may corrode, releasing ions into solution. ${ }^{(2)}$

This process will continue until equilibrium is reached, or until some occurrence impedes ion release. ${ }^{(3,4)}$ For example, passivating film may form to prevent the metal from coming into contact with the solution. The surfaces of all metals react with oxygen to form a surface oxide layer, which inhibits an attacking substance from reaching the metal surface.

Metals and alloys that rely on a passive film for corrosion resistance all share the property that, at sufficiently high potentials, the passive layer can be broken down and the metal or alloy will no longer be protected. ${ }^{(5,6)}$ Most of those mentioned factors are highly dependent on the shaping process, so changing the cross section shape does not have a direct influence on the total surface area only but also on those parameters. Accordingly our aim from this study was originated.

Therefore, in this study in vitro ion release from rectangular and round cross sectional wires made of several alloys was carried out to investigate the influence of material composition as well as the shape on the ion release.

\section{MATERIAL AND METHOD}

Orthodontic wires with rectangular $(0.016 \times 0.022$ inch $)$ and round (0.018 inch) cross section made of stainless steel, nickel-titanium, 
Egyptian

Orthodontic Journal

Nickel-copper-titanium alloys were purchased (Ormco, USA). In addition the upper central incisor brackets were selected (American Orthodontics, USA). A fluid was used as electrolyte. This latter was the commercially available normal saline $(0.9 \mathrm{NaCl})$.

Simulating the oral environment, the mesh area of the bracket was isolated by composite (greengloo, Two-way color change adhesive, Ormco, USA) .Composite assured isolation of this specific area since the experiments were concerned only with the wire and the surface area of the bracket slot.

The orthodontic wires were fixed onto the brackets using elastomeric ties (o-Ties). The wire/bracket couples were immersed in separate glass tubes containing the simulated human saliva. The amount of the used fluid was chosen to keep the solid (wire/bracket couples) to fluid ratio 1:50 by weight. The glass tube had been incubated at $37 \pm 1{ }^{\circ} \mathrm{C}$ for three successive weeks. Within specific time intervals (every week), the samples were withdrawn from the incubator. Wires were separated from the brackets then washed many times carefully using distilled water and acetone and vacuously dried for 30 seconds and then weighed.

This was done to avoid any effect of the water sorption by the composite which can affect the weight. In addition, it was not possible to standardize the thickness of the composite layer as well as its weight. It has to be noted that weight loss was calculated in grams (gm).

Moreover, $3 \mathrm{ml}$ samples were withdrawn from the fluid to check the ion content by atomic absorption spectroscopy (A Analyst Absorption Spectroscopy 100/300 Atomic Absorption Spectrometer). It is noteworthy mentioned that, after withdrawing the samples, the fluid was compensated to keep the solid fluid ratio in each glass tube constant. Ion release was calculated by part per billion (ppb).

The specimens were given the following codes:

S: Stainless steel, NT: NiTi alloy, CNT: CUNiTi, R: round cross section, $\mathrm{S}$ (before the number): Rectangular cross section and finally the number represented the week of incubation. As example, NTR1 stands for rounded Nickel-Titanium wire after one week of incubation. Concerning statistical analysis, Student's t-test hypothesis was utilized to validate the results. 


\section{RESULTS}

Fig 1 shows the defect of the incubation time on the weight of the utilized orthodontic wires (round and rectangular). Fig 1A represents the relationship between the percentage weight change in round wires and the incubation time. An increase in the wires weights was detected in case of stainless steel wires. For the other two alloys: nickel-titanium and nickelcopper-titanium, a non-significant weight change was observed.

Viewing rectangular wires (Fig 1B), there was almost no weight change in stainless steel wires. NiTi wires showed a good performance concerning weight change. This performance is lesser than stainless steel wires. However, the difference between the two wires is statistically nonsignificant. Concerning nickel-copper-titanium wire, an increase in weight followed by a decrease was detected.

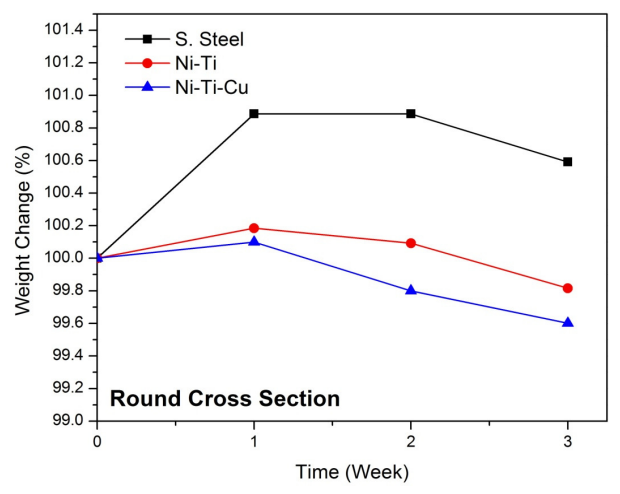

Fig 1A

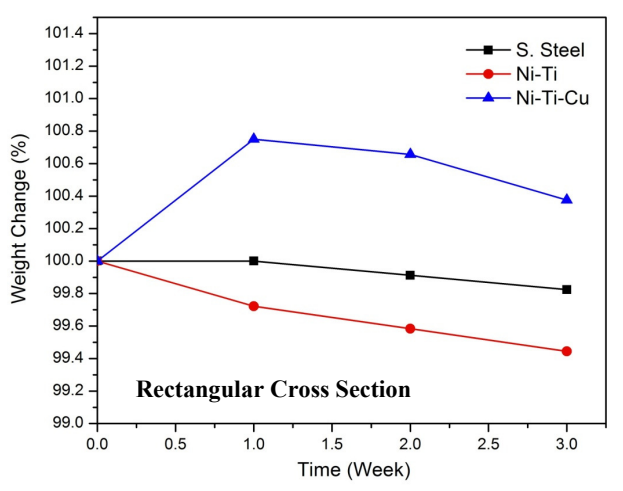

Fig 1B

Fig.1: Effect of incubation time on the weight of the utilized orthodontic wires; (A) round cross section wires and (B) rectangular cross section ones.

Comparing round and rectangular cross section results, it can be denoted that there was no distinct influence on the nickel- titanium alloy. There was no significant difference on the behavior of this alloy in the two investigated shapes.

To properly figure out the cross section shape effect in cases of stainless steel and nickel-copper-titanium wires, the corresponding data was separately demonstrated in fig 2 . It is noteworthy mentioning that the same scale was used in both figures. 


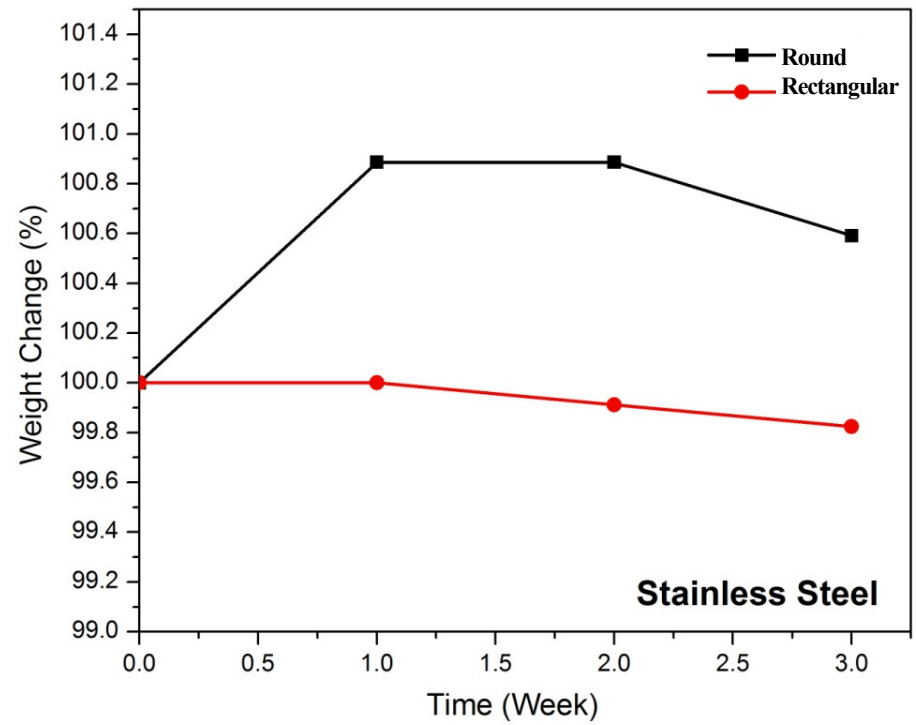

Fig 2A

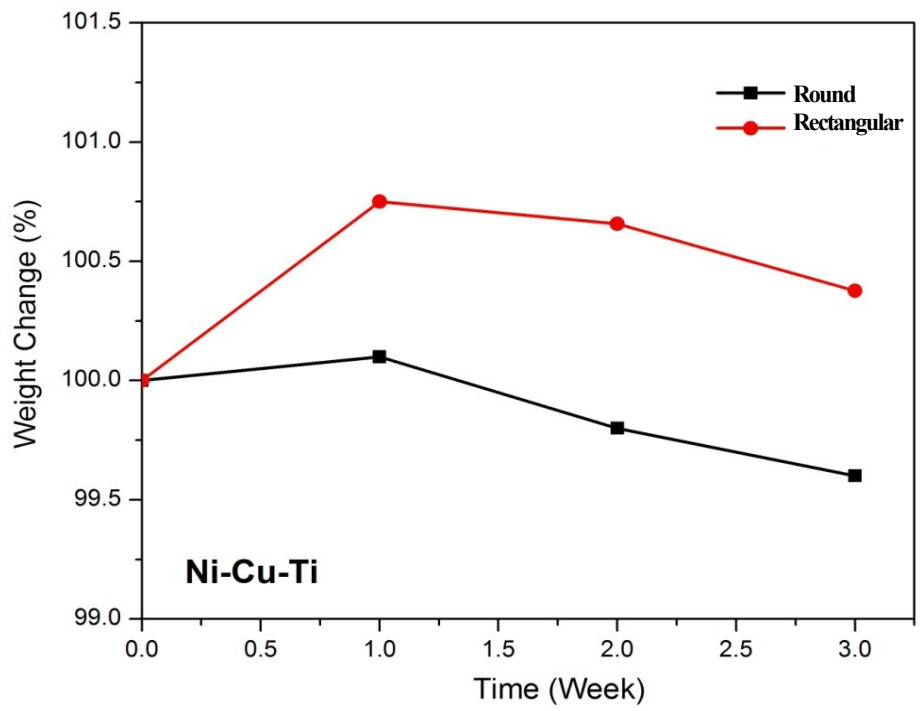

Fig 2B

Fig. 2: Effect of cross section morphology on the weight change in case of (A) stainless steel orthodontic wires; and (B) Ni-Cu-Ti ones. 
The effect of incubation time on the iron (Fe) ion (Fig 3A) and chromium $(\mathrm{Cr}$ ) ions (Fig 3B) concentrations in case of round cross section wires compared with the rectangular cross section ones which support the results obtained in fig 1 and 2A indicating that rectangular cross section stainless steel wires showed good performance compared with round cross sections ones.

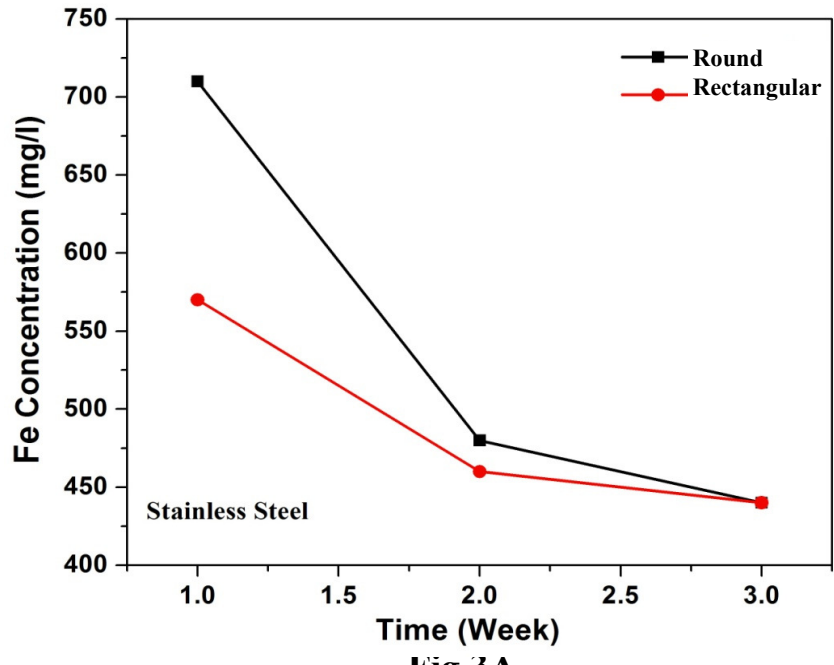

Fig 3A

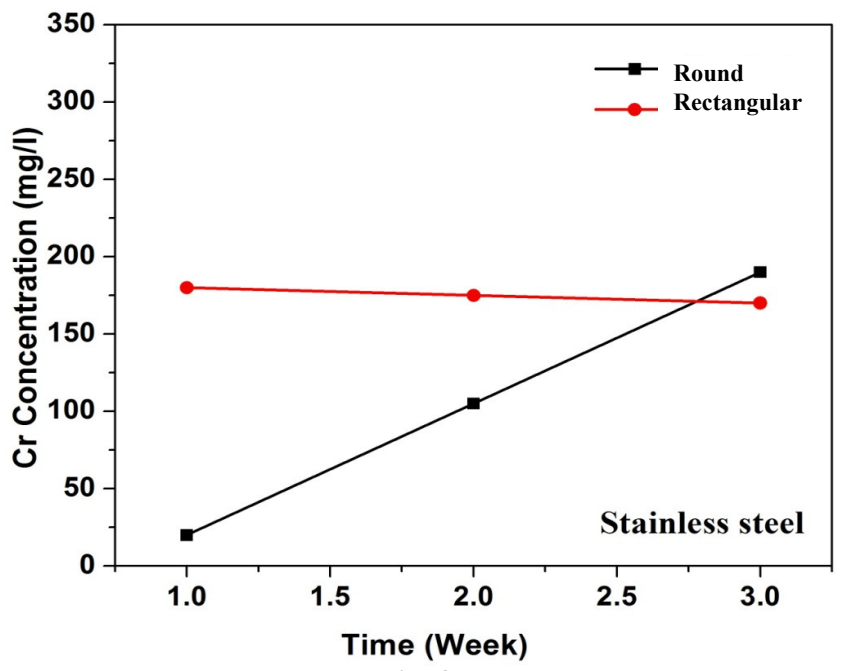

Fig 3B

Fig 3: Effect of incubation time on release of $\mathrm{Fe}$; (A) and $\mathrm{Cr}$; (B) ions in case of using stainless steel orthodontic wires. 
With time passing, $\mathrm{Fe}$ ion concentration in both morphologies decreased. As shown in fig 3B, Cr ion concentration in the soaking fluid did not change after the first week. However, in case of round wires, $\mathrm{Cr}$ concentration did have direct relationship with the incubation time which indicated continuous corrosion in such wires.

The iron release behavior in case of soaking $\mathrm{Ni}-\mathrm{Cu}-\mathrm{Ti}$ orthodontic wires with round and rectangular cross sections is represented in Fig 4. The change of $\mathrm{Cu}$ concentration with incubation time (Fig 4A), $\mathrm{Cu}$ ions concentration increased with the increase of incubation time in both round and rectangular wires.

Analogy of $\mathrm{Fe}$ ion in case of stainless steel wires (Fig 3A) was obtained when Ni ions were investigated in the utilized fluid in case of $\mathrm{Ni}-\mathrm{Cu}-\mathrm{Ti}$ alloy as shown in fig 4B.

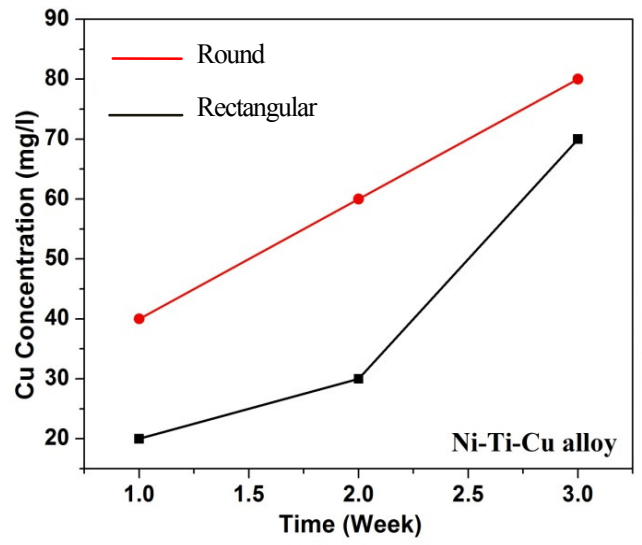

Fig 4A

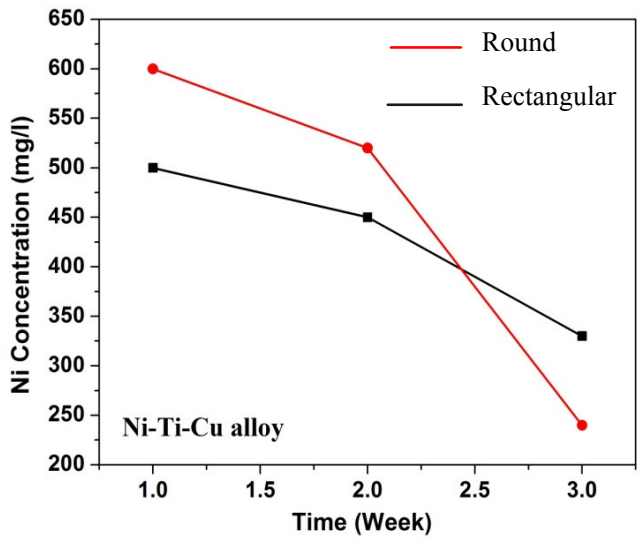

Fig 4B

Fig.4: Effect of incubation time on release of $\mathrm{Cu}(\mathrm{A})$ and $\mathrm{Ni}(\mathrm{B})$ ions in case of using $\mathrm{Cu}-\mathrm{Ni}$-Ti orthodontic wires.

The effect of the incubation time on $\mathrm{Cr}$ concentration in the utilized simulated human saliva fluid was also studied (Fig 4C). As the Ni-Cu-Ti orthodontic wires do not have $\mathrm{Cr}$ element, the $\mathrm{Cr}$ ion detected in the fluid was released from the stainless steel brackets. Cr concentration increased with the increase of the incubation time. However, more ions were obtained in case of rectangular wires. 


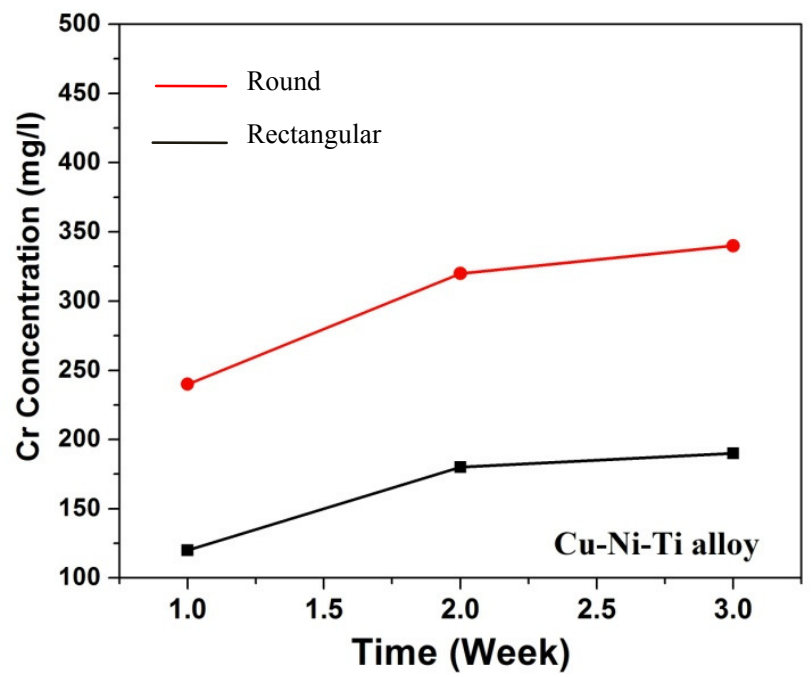

Fig 4C: Effect of incubation time on release of $\mathrm{Cr}$ ions in case of using $\mathrm{Cu}-\mathrm{Ni}-\mathrm{Ti}$ orthodontic wires.

\section{DISCUSSION}

The corrosion behavior of dental alloys is of great interest as an indication of their biocompatibility. It occurs in aqueous solutions, on the surface, as electrochemical reactions which contain free electrons. These reactions are continuous resulting in dissociation of metal or loss of ions. ${ }^{(7,8)}$

In orthodontics, a broad spectrum of stainless steel and titanium based alloys are used as archwires and brackets. From these alloys, different metallic ions are released. The major ones include $\mathrm{Fe}, \mathrm{Cr}$, $\mathrm{Ti}$, and Ni. Nickel discharge has a strong immunological effect and may result in hypersensitivity, contact dermatitis and cytotoxicity. ${ }^{(9)}$ Nickel and chromium received the most attention due to their reported potential for producing allergic, toxic or carcinogenic effects. ${ }^{(10,11)}$

Although of the tremendous researches about ion release in the mouth due to orthodontic treatment, the effect of wire cross section was not given the interest it deserves. Not only the cross section but also the loss of weight has not to be overlooked. Accordingly, this study was carried out to highlight the effect of cross section on ion release and 
subsequent weight loss from three different alloys used in orthodontics: stainless steel, nickel-titanium and copper- nickel-titanium.

The specimens (wire/bracket couple) constituted of upper incisor brackets, to profit from its minimum curvature and larger contact area with the wire, as well as wires $(0.018$ round or $0.016 \mathrm{x} 0.022$ rectangular) attached by elastomeric ties. The mesh surface was isolated by composite.

Stainless steel brackets were used due to their popularity. It was thought that using the same kind of brackets in all experiments did not affect the general drawn conclusion in our study because if the brackets release some ions it will be the same in all cases.

From the various method of studying ion release, weighing the couple alloy before and after immersion in the corrosive solution and using atomic absorption spectroscopy were valid. ${ }^{(8)}$

In the present study, a normal saline solution $(0.9 \% \mathrm{Na} \mathrm{Cl})$ was used because $\mathrm{Na} \mathrm{Cl}$ is a corrosive agent. The solution temperature was controlled at $37^{\circ} \mathrm{C}$ with thermostat to simulate the oral condition. This temperature $\left(37^{\circ} \mathrm{C}\right)$ represents normal temperature of oral-cavity ${ }^{(12)}$. Previous study recommended that corrosion testing of dental material should be carried out at $37^{\circ} \mathrm{C}$ as standard temperature ${ }^{(13)}$.

The static immersion tests were carried out at specific periods ( $7,14,21$ days). At the end of each period, the specimens were removed. The effect of incubation time on the wire (round and rectangular) weight as well as on the ion release was studied.

Viewing change in weight, increase in wires weight was detected. This can be attributed to the formation of the metal oxide layers (passive layer) on the wires surfaces. ${ }^{(14,15,16,17)}$ Therefore, one can say that more increase in the wire weight may be explained as more metal oxide was formed on the surface which indicates that low corrosion resistance of that wire.

Round stainless steel wires did have the lowest corrosion resistance as they demonstrated high change in the weight compared to round $\mathrm{NiTi}$ and $\mathrm{CuNiTi}$ wires. However, the weight of metal oxides layer on the stainless steel wires almost did not change after the first week. 
Rectangular stainless steel wires showed better performance compared to round ones. Conversely, the rectangular CuNiTi wires exhibited least performance concerning weight loss. This can be explained as Niti wires also form a passive layer consisting of several oxides of $\mathrm{TiO}_{2}, \mathrm{TiO}$ and $\mathrm{Ti}_{2} \mathrm{O}_{5}$ which provide the good biocompatibility of the NiTi alloy. This was proved by many researches. ${ }^{(18,19,20)}$

No doubt that the wires with rectangular cross section do have higher total surface area per unit length than those having round cross section area when the rectangular diagonal equals the circle diameter. However, our aim in this study does not relate to the total surface area but to the other factors coming from changing the shape.

To properly investigate the corrosion behavior of the utilized orthodontic wires, the concentration of some specific ions in the soaking fluid had been also determined. The release of $\mathrm{Fe}, \mathrm{Cu}, \mathrm{Ni}$ and $\mathrm{Cr}$ was inspected.

Although isolation of the mesh area (apparently of the base) does not imply isolation of the buccal area of the bracket base as well, which may be implicated in the corrosion process along with the wire and the bracket slot. The study tried to simulate the situation inside the oral cavity. Accordingly, the derived conclusions will not be affected by the release of some ions from the labial surface or even the bracket slot.

Depletion in $\mathrm{Fe}$ ion concentration was detected. This can be attributed to the formation of iron hydroxide precipitate which reduces the $\mathrm{Fe}$ ions in the solution. The results are in accordance with those of Hunt $(1999)^{(21)}$ and Keun-taek et al (2002). ${ }^{(22)}$

Concerning $\mathrm{Cr}$ ions release in the soaking solution, the result obtained support our conclusion saying that rectangular stainless steel wires corrode less than round ones. It has to be noticed, when inspecting $\mathrm{Cr}$ ion concentration in case of using CuNiTi wires, that these wires do not have chromium element in their composition. Accordingly the detected $\mathrm{Cr}$ ion in the solution is released from the stainless steel brackets.

As same as $\mathrm{Fe}$, Ni concentration decreased with incubation time. This is due to the formation of nickel hydroxide precipitate. Moreover, 
the results support our previous conclusion about CuNiTi wires. The figures can be considered as additional evidence for recommending using CuNiTi with round cross section rather than rectangular one.

\section{CONCLUSIONS}

1- NiTi wires have the lowest corrosion rate (highest corrosion resistance) compared to stainless steel and $\mathrm{CuNiTi}$ wires. In addition the wire cross section has a negligible effect.

2- In case of stainless steel wires, the rectangular wires revealed better performance compared to round ones.

3- CuNiTi wires have the least corrosion resistance manifested by weight loss and ion release. However, round wires showed better performance compared to rectangular ones.

4- An increase in the wires weights was detected in case of stainless steel wires. For the other two alloys: nickel-titanium and nickel-coppertitanium, a non-significant weight change was observed.

5- In case of rectangular copper-titanium, weight loss was observed

\section{REFERENCES}

1- Rondelli $\mathrm{H}$ and Vicentini B: Localized corrosion behavior in simulated human body fluids of commercial Ni-Ti orthodontic wires. Biomaterials; 1999, 20: 785-792.

2- Huang H H, Chiu Y H, Lee T H, Wu S C, Yang H W, Su K H, and Hsu C C: Iron release from NiTi orthodontic wires in artificial saliva with various acidities. Biomaterials, 2003, 34: 3585-3592.

3- Bertrand A L, Morrier J J, Benay G, Ponosonnet L and Barsotti O: Effects of various hygienic treatments of orthodontic alloys on in vitro mutans streptococci adherence. Euro. Cells Mater; 2005, 9: 39-40.

4- Tan L, Dod R A and Crone W C: Corrosion and wear- corrosion behavior of Niti modified by plasma source ion implantation. Biomaterials; 2003, 24, 2931-2939.

5- Staffolani N, Damiani F, Lilli C, Guerra M, Staffolani N J, Belcastro and Locci P: Ion release from orthodontic appliances. J of Dentistry; 1999, 27: 449-454. 
6- Kim H and Johnston J W: Corrosion of stainless steel, nickel-titanium, coated nickel-titanium and titanium orthodontic wires. The Angle Orthodontist; 1999, 69 (1): 39-44.

7- Kao CT, Huang TH.: Variations in surface characteristics and corrosion behavior of metal brackets and wires in different electrolyte solutions. Eur J Orthod. 2010 Oct;32(5):555-60

8- Al-Hity R, Kappert H, Viennot S, Dalard F, Grosgogeat B: Corrosion resistance measurements of dental alloys, are they correlated" Dental Materials,(2007) 23, Issue 6, Pages 679-687

9- Hwang CJ, Shin JS, Cha JY: Metal release from simulate fixed orthodontic appliances. Am J Orthod \& Dentofac Orthop (2001); 120:383-391.

10- McKay G.C., Macnair R., MacDonald C. Grant M.H. Interaction of orthopeadic metals with an immortalized rat osteoblast cell. Biomaterials 17 (1996); 1339-44.

11- Maruthamuthu S, Rajasekar A, Sathiyanarayanan S, Muthukumar N and Palaniswamy N. Electrochemical behavior of microbes on orthodontic wires. Current science (2005); 89 (6) 25: 988-996.

12- Olsson S., Berglund A, Bergman: Release of elements due electrochemical corrosion of dental amalgam. J. Dent, Res. (1994);73, p 33 -43

13- Kedic SA, Abbas Aksut , Ali Kilicarslan M. Bayramoglu G., Gokdemirk: Corrosion Behavior of dental metals and alloys in different media. J. Oral Rehab (1998); $25,800-8$

14- El Medawar L, Rocher P, Hornez JC, Traisnel M, Breme J, Hildebrand HF. "Electrochemical and cytocompatibility assessment of NiTiNOL memory shape alloy for orthodontic use. Biomol Eng. (2002);19(2-6):153-60.

15- Rondelli G.; Vicentini B: Localized corrosion behavior in simulated human body fluids of commercial Ni-Ti orthodontic wires. Biomat (1999).(20),8;785-792

16- Speek KM, Fraker AC: Anodic polarization behavior of NiTi and Ti6A14V in simulated physiological solutions. J Dent Res 1980; (59): 1590-95. 
17- Sedriks AJ, Green JA, and Novak DL: Electrochemical behavior of NiTi alloys in acidic chloride solutions. Corrosion; 28 (1972) 137-142.

18- Peitsch T,Klocke A, Kohl Nieke B, prymak O, Epple M: The release of Nickel from orthodontic NiTi wires in increased by dynamic mechanical loading but not constrained by surface nitridation. J.Biomed.Mater Res. 82A (2007); 731- 739

19- Lin MC, Lin SC, Lee TH, Huang HH: surface analysis and corrosion resistance of different stainless steel orthodontic brackets in artificial saliva. Angle orthodontist 2006; (76) 322-329

20- Her- Hsiung: Surface characterization and corrosion resistance of Nickel- Titanium orthodontic archwires in artificial saliva of various degree of acidity. J. Biomed. Mater Res. 74A (2005) 629- 639

21- Hunt N.P, Cunningham SJ, Golden CG, Sheriff M: An investigation into the effects of polishing on surface hardness and corrosion of orthodontic archwires. Angle Orthod.1999; Oct; 69(5):433-40.

22- Keun-Taek Oh, Chung-Ju Hwang, Yong-Soo Park, and Kyoung-Nam Kim: In Vitro Corrosion Resistance of Orthodontic Super Stainless Steel Wire. J. Electrochem. Soc 2002; (149),9; B387-B392

23- John W. Edie, George F. Andreasen \& Mary P. Zaytoun: Surface Corrosion of Nitinol and Stainless Sleet Under Clinical Conditions. Angle orthodontics 1981; (51)4,319-24.

24- Petoumenou E, Arndt M, Keilig L, Reimann S, Hoederath H, Eliades T, Jäger A, Bourauel C: Nickel concentration in the saliva of patients with nickel-titanium orthodontic appliances. Am J Orthod Dentofacial Orthop. 2009; Jan; 135(1):59-65.

25- Suárez C, Vilar T, Gil J, Sevilla P.: In vitro evaluation of surface topographic changes and nickel release of lingual orthodontic archwires. J Mater Sci Mater Med. 2010; Feb;21(2):675-83 